\title{
Challenges and Opportunities for Efficient Computing with FAWN
}

\author{
Vijay Vasudevan, David G. Andersen, Michael Kaminsky*, Jason Franklin \\ Michael A. Kozuch*, Iulian Moraru, Padmanabhan Pillai*, Lawrence Tan \\ Carnegie Mellon University, ${ }^{*}$ Intel Labs Pittsburgh
}

\begin{abstract}
This paper presents the architecture and motivation for a clusterbased, many-core computing architecture for energy-efficient, dataintensive computing. FAWN, a Fast Array of Wimpy Nodes, consists of a large number of slower but efficient nodes coupled with low-power storage. We present the computing trends that motivate a FAWN-like approach, for CPU, memory, and storage. We follow with a set of microbenchmarks to explore under what workloads these FAWN nodes perform well (or perform poorly), and briefly examine scenarios in which both code and algorithms may need to be re-designed or optimized to perform well on an efficient platform. We conclude with an outline of the longer-term implications of FAWN that lead us to select a tightly integrated stacked chipand-memory architecture for future FAWN development.
\end{abstract}

\section{Categories and Subject Descriptors}

D.4.7 [Operating Systems]: Organization and Design-Distributed Systems; D.4.2 [Operating Systems]: Storage Management; D.4.8 [Operating Systems]: Performance-Measurements

\section{General Terms}

Performance, Experimentation, Measurement

\section{Keywords}

Design, Energy Efficiency, Performance, Measurement, Cluster Computing, Flash

\section{INTRODUCTION}

Power is becoming an increasingly large financial and scaling burden for computing. The direct and related cost of power in large data centers is a growing fraction of their cost-up to $50 \%$ of the three-year total cost of owning a computer - to the point that companies such as Microsoft, Google, and Yahoo! have built new data centers close to large and cost-efficient hydroelectric power sources [13]. Datacenter density is limited by their ability to supply and cool $10-20 \mathrm{~kW}$ of power per rack and up to 10-20 MW per datacenter [17]. Future datacenters may require as much as $200 \mathrm{MW}$ [17], and today, datacenters are being constructed with dedicated electrical substations to feed them. While

Brands and names are the property of their respective owners. power constraints have pushed the processor industry toward multicore architectures, energy-efficient alternatives to traditional disk and DRAM-based cluster architectures have been slow to emerge.

As an energy-efficient alternative for data-intensive computing, we present a cluster architecture called a Fast Array of Wimpy Nodes, or FAWN. A FAWN consists of a large number of slower but efficient nodes that each draw only a few watts of power, coupled with low-power storage. We have explored prototype FAWN nodes ranging from five-year old, $500 \mathrm{MHz}$ embedded devices using CompactFlash storage, to more modern Intel Atom-based nodes with fast solid-state drives.

In this paper, we describe the long-lasting, fundamental trends in the scaling of computation and energy that suggest that the FAWN approach will become suitable for increasing classes of workloads. First, as we show in Section 2, slower, simpler processors can be more efficient: they use fewer joules of energy per instruction than higher speed processors. Second, dynamic power scaling techniques are less effective than reducing a cluster's peak power consumption. After examining CPU scaling trends, we similarly examine the same scaling questions for both memory capacity/speed and for storage.

We then summarize our experience with real FAWN architectures for a variety of workloads: seek-bound, I/O-throughput bound, memory-bound, and CPU-bound. FAWN can be several times more efficient than traditional systems for I/O-bound workloads, and on par with or more efficient for many memory and CPU-limited applications (Section 3).

Our experiences highlight several challenges to achieving the potential energy efficiency benefits of the FAWN approach. Existing software may not run as well on FAWN nodes which have limited resources (e.g., memory capacity, CPU cache sizes); achieving good performance often requires new algorithms and optimizations. Existing low-power hardware platforms have high fixed power costs that diminish the potential efficiency returns. We explore these issues in Sections 2 and 3. We conclude with a future vision for FAWN-like hardware by exploring the construction of low-GHz, many-core systems for data-intensive applications in Section 4.

\section{COMPUTING TRENDS}

The FAWN approach to building well-matched cluster systems has the potential to achieve high performance and be fundamentally more energy-efficient than conventional architectures for serving massive-scale I/O and data-intensive workloads. We measure system performance in work done per second and measure energyefficiency in work done per Joule (equivalently, performance per Watt). FAWN is inspired by several fundamental trends: 


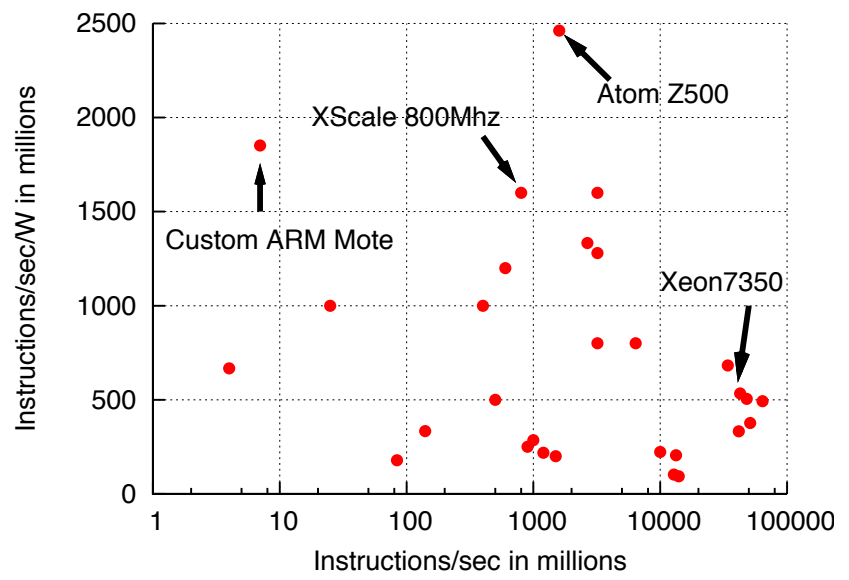

Figure 1: Max speed (MIPS) vs. Instruction efficiency (MIPS/W) in log-log scale. Numbers gathered from publiclyavailable spec sheets and manufacturer product websites as of 2009.

Increasing CPU-I/O Gap Over the last several decades, the gap between CPU performance and I/O bandwidth has continually grown. For data-intensive computing workloads, storage, network, and memory bandwidth bottlenecks often cause low CPU utilization.

FAWN Approach: To efficiently run I/O-bound data-intensive, computationally simple applications, FAWN uses lower frequency, simpler processors selected to reduce I/O-induced idle cycles while maintaining high performance. The reduced processor speed then benefits from a second trend:

CPU power consumption grows super-linearly with speed Operating processors at higher frequency requires more energy, and techniques to mask the CPU-memory bottleneck come at the cost of energy efficiency. Branch prediction, speculative execution, outof-order execution and increasing the amount of on-chip caching all require additional processor die area; modern processors dedicate as much as half their die to L2/3 caches [15]. These techniques do not increase the speed of basic computations, but do increase power consumption, making faster CPUs less energy efficient.

FAWN Approach: A FAWN cluster's slower CPUs dedicate more transistors to basic operations. These CPUs execute significantly more instructions per Joule than their faster counterparts (Figure 1): multi-GHz superscalar quad-core processors can execute approximately 100 million instructions per Joule, assuming all cores are active and avoid stalls or mispredictions. Lower-frequency inorder CPUs, in contrast, can provide over 1 billion instructions per Joule - an order of magnitude more efficient while still running at $1 / 3$ rd the frequency.

Worse yet, running fast processors below their full capacity draws a disproportionate amount of power:

Dynamic power scaling on traditional systems is surprisingly inefficient A primary energy-saving benefit of dynamic voltage and frequency scaling (DVFS) was its ability to reduce voltage as it reduced frequency [30], but modern CPUs already operate near minimum voltage at the highest frequencies.

Even if processor energy was completely proportional to load, non-CPU components such as memory, motherboards, and power supplies have begun to dominate energy consumption [4], requiring

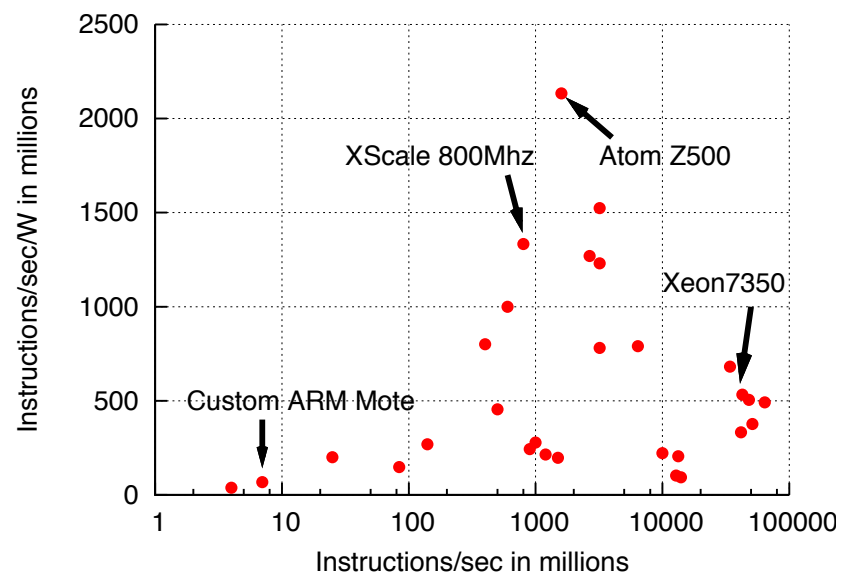

Figure 2: Processor efficiency when adding $0.1 \mathrm{~W}$ of fixed system overhead.

that all components be scaled back with demand. As a result, running a modern, DVFS-enabled system at $20 \%$ of its capacity may still consume over $50 \%$ of its peak power [28]. Despite improved power scaling technology, systems remain most energy-efficient when operating at peak utilization. Given the difficulty of scaling all system components, we must therefore consider "constant factors" for power when calculating a system's instruction efficiency. Figure 2 plots processor efficiency when adding a fixed $0.1 \mathrm{~W}$ cost for basic system components such as $10 \mathrm{Mbps}$ Ethernet (e.g., the Intel $82552 \mathrm{~V}$ consumes $59 \mathrm{~mW}$ at idle). Because these overheads dwarf the CPU power draw of tiny sensor-type processors that consume only micro-Watts, their efficiency as a cluster node drops significantly. The best operating point exists in the middle of the curve, where the fixed costs are amortized while still providing energy efficiency.

The $0.1 \mathrm{~W}$ fixed overhead in Figure 2 demonstrates that even a tiny amount of power draw can reduce the efficiency of extremely slow processors. In practice, these "fixed" costs vary depending on the platform. For example, a high end server processor would need a higher speed network with higher power draw to balance its processing capabilities, and so the fixed costs would be different than those for a slower balanced system.

The important lesson is that system designers must choose the processor that takes into account the unavoidable fixed costs of the rest of the system, and must engineer away the avoidable fixed costs. The system that best eliminates these avoidable fixed costs in relation to its processing capability will therefore see gains in energy efficiency. For example, SeaMicro's SM10000 consists of a custom networking fabric to connect its 512 Intel Atom nodes to amortize the power cost of networking in comparison to the processor power draw [25].

Newer techniques aim for energy proportionality by turning machines off and using VM consolidation, but the practicality of these techniques is still being explored. Many large-scale systems often operate below $50 \%$ utilization, but opportunities to go into deep sleep states are few and far between [4], while "wake-up" or VM migration penalties can make these techniques less energy-efficient. Also, VM migration may not apply for some applications, e.g., if datasets are held entirely in DRAM to guarantee fast response times. 
Even if techniques for dynamically scaling below peak power were effective, operating below peak power capacity has one more drawback:

Peak power consumption limits data center density Data centers must be provisioned for a system's maximum power draw. This requires investment in infrastructure, including worst-case cooling requirements, provisioning of batteries for backup systems on power failure, and proper gauge power cables. FAWN significantly reduces maximum power draw in comparison to traditional cluster systems that provide equivalent performance, thereby reducing infrastructure cost, reducing the need for massive over-provisioning, and removing one limit to the achievable density of data centers.

Finally, energy proportionality alone is not a panacea: systems ideally should be both proportional and efficient at $100 \%$ load. In this paper, we show that there is significant room to improve energy efficiency, and the FAWN approach provides a simple way to do so.

\subsection{Memory trends}

The previous section examined the trends that cause CPU power to increase drastically with an increase in sequential execution speed. In pursuit of a balanced system, one must ask the same question of memory and storage as well.

Understanding DRAM power draw DRAM has, at a high level, three major categories of power draw:

Idle/Refresh power draw: DRAM stores bits in capacitors; the charge in those capacitors leaks away and must be periodically refreshed (the act of reading the DRAM cells implicitly refreshes the contents). As a result, simply storing data in DRAM requires nonnegligible power.

Precharge and read power: The power consumed inside the DRAM chip. When reading a few bits of data from DRAM, a larger line of cells is actually precharged and read by the sense amplifiers. As a result, random accesses to small amounts of data in DRAM are less power-efficient than large sequential reads.

Memory bus power: A significant fraction of the total memory system power draw-perhaps up to $40 \%$ - is required for transmitting read data over the memory bus back to the CPU or DRAM controller.

Design tradeoffs Designers can somewhat improve the efficiency of DRAM (in bits read per joule) by clocking it more slowly, for the same reasons mentioned for CPUs. In addition, both DRAM access latency and power grow with the distance between the CPU (or memory controller) and the DRAM: without additional amplifiers, latency increases quadratically with trace length, and power increases at least linearly. This effect creates an intriguing tension for system designers: Increasing the amount of memory per CPU simultaneously increases the power cost to access a bit of data. The reasons for this are several: To add more memory to a system, desktops and servers use a bus-based topology that can handle a larger number of DRAM chips; these buses have longer traces and lose signal with each additional tap. In contrast, the low-power DRAM used in embedded systems (cellphones, etc.), LPDDR, uses a pointto-point topology with shorter traces, limiting the number of memory chips that can be connected to a single CPU, and reducing substantially the power needed to access that memory.

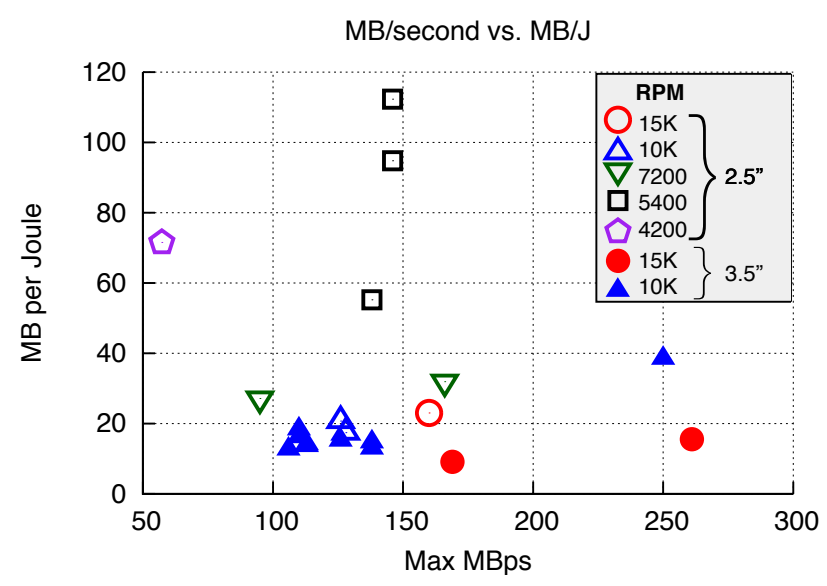

Figure 3: Power increases with rotational speed and platter size. Solid shapes are 3.5" disks and outlines are 2.5" disks. Speed and power numbers acquired from product specification sheets.

\subsection{Storage Power Trends}

The energy draw of magnetic platter-based storage is related to several device characteristics, such as storage bit density, capacity, throughput, and latency. Spinning the platter at faster speeds will improve throughput and seek times, but requires more power because of the additional rotational energy and air resistance. Capacity increases follow bit density improvements and also increase with larger platter sizes, but air resistance increases quadratically with larger platter sizes, so larger platters also require more power to operate.

Figure 3 demonstrates this tradeoff by plotting the efficiency versus speed for several modern hard drives, including enterprise, mobile, desktop, and "Green" products. ${ }^{1}$ The fastest drives spin at between 10-15K RPM, but they have a relatively low energy efficiency as measured by MB per Joule of max sustained sequential data transfer. The 2.5 " disk drives are nearly always more energy efficient than the 3.5" disk drives. The most efficient drives are 2.5 " disk drives running at 5400 RPM. Energy efficiency therefore comes at the cost of per-device storage capacity for magnetic hard drives.

Our preliminary investigations into flash storage power trends indicate that the number of IOPS provided by the device scales roughly linearly with the power consumed by the device, likely because these devices increase performance through chip parallelism instead of by increasing the speed of a single component.

\section{WORKLOADS}

In this section, we describe under what conditions a FAWN architecture can provide superior energy efficiency, and where traditional architectures can be as efficient, or in some cases, more energyefficient than low-power systems.

\footnotetext{
${ }^{1}$ The figure uses MB/s data from vendor spec sheets, which are often best-case outer-track numbers. The absolute numbers are therefore somewhat higher than what one would expect in typical use, but the relative performance comparison is likely accurate.
} 


\subsection{Metrics}

Evaluating large systems using only performance metrics such as throughput or latency is slowly falling out of favor as energy and space constraints inform the design of modern large scale systems. There are several metrics for energy efficiency, but the one we focus on is "work done per Joule" of energy, or equivalently, "performance per Watt."

Low-power VLSI designs have alternatively looked at the "energy-delay product," which multiplies the amount of energy to do an amount of work with the time it takes to do that amount of work. This penalizes solutions that reduce the amount of energy by reducing performance for energy efficiency gains. Others have gone further by proposing using "energy delay ${ }^{2}$ " to further penalize solutions that simply reduce voltage at the expense of performance.

However, for large-scale cluster computing applications that are consuming a significant fraction of energy in datacenters worldwide, "work done per Joule" is an appropriate metric. This metric relies on being able to parallelize workloads, which is often explicitly provided by data-intensive computing models such as MapReduce [10] that harness data-parallelism.

More specifically, when the amount of work is fixed but parallelizable, one can use a larger number of slower machines yet still finish the work in the same amount of time-for example, ten nodes running at one-tenth the speed of a traditional node. If the aggregate power used by those ten nodes is less than that used by the traditional node, then the ten-node solution is more energy-efficient.

\subsection{Taxonomy}

We begin with a broad classification of the types of workloads found in data-intensive computing whose solution requires largescale datacenter deployments:

1. I/O-bound workloads

2. Memory/CPU-bound workloads

3. Latency-sensitive, but non-parallelizable workloads

4. Large, memory-hungry workloads

The first of these workloads, I/O-bound workloads, have running times that are determined primarily by the speed of the $\mathrm{I} / \mathrm{O}$ devices (typically disks for data-intensive workloads). I/O-bound workloads can be either seek- or scan-bound, and represent the lowhanging fruit for the FAWN approach, as described in our earlier work [2]. In the next sections, we discuss two examples of I/Obound workloads: key-value storage and large sorts.

The second category includes CPU and memory-bound workloads, where the running time is limited by the speed of the CPU or memory system. The last two categories represent workloads where the FAWN approach may be less useful. Latency-sensitive workloads require fast responses times to provide, for example, an acceptable user-experience; anything too slow (e.g., more than 50ms) impairs the quality of service unacceptably. Finally, large, memoryhungry workloads frequently access data that can reside within the memory of traditional servers (on the order of a few to $10 \mathrm{~s}$ of gigabytes per machine today). As we describe in Section 3.6.2, the data structure created in $g r e p$ when searching for millions of short phrases requires several gigabytes of memory and is accessed randomly. This causes frequent swapping on FAWN nodes with limited memory, but fits entirely in DRAM on modern servers.

\begin{tabular}{lccc} 
System / Storage & QPS & Watts & $\frac{\text { Queries }}{\text { Joule }}$ \\
\hline $\begin{array}{l}\text { Embedded Systems } \\
\text { Alix3c2 / Sandisk(CF) }\end{array}$ & 1298 & 3.75 & 346 \\
\hline Modern Systems & & & \\
Server i7 / Fusion-io & 61494 & 194 & 317.0 \\
Desktop i7 / X25-E (x6) & 59448 & 98.0 & 606.6 \\
Atom / X25-E & 10760 & 22.3 & 482.5
\end{tabular}

Table 1: Query performance and efficiency for different machine configurations. The Atom node is a prototype.

\subsection{Key-value Workload}

Our prior work proposed the Fast Array of Wimpy Nodes (FAWN) architecture, which uses a large number of FAWN nodes that act as data storage/retrieval nodes [2]. These nodes use energyefficient, low-power processors combined with low-power storage and a small amount of DRAM. We compare FAWN-type systems with traditional architectures to understand which system is more energy-efficient in terms of work done per Joule. For all experiments where we measure energy efficiency, we use a "Watts Up?" power meter that integrates power draw at the wall socket and reports the power consumed in Watts once per second [29]. We calculate the number of Joules consumed during the course of each experiment to compute energy efficiency values, and report the average power draw during the course of the experiment where appropriate.

Table 1 presents an update of the exploration that we began in our previous work. It shows the rate at which various node configurations can service requests for random key-value pairs (1 KB values) from an on-disk dataset, via the network. When we began this work over two years ago, the best embedded system (Alix3c2) using CompactFlash (CF) storage was six times more power-efficient (in queries/joule) than a 2008-era low-power desktop equipped with a contemporary SATA-based flash device (see [2] for these numbers).

Since our initial exploration, however, the low-power server market has expanded dramatically. We recently benchmarked several modern systems to understand which platform can provide the highest queries per Joule for persistent key-value storage. We have included in our comparisons three different systems that all use modern flash devices. At the high-end server level (Server i7), we use a dual-socket quad-core, rackmount Intel Core i7 (Nehalem) processor system with 16 GB of DRAM and an 80 GB Fusion-io ioDrive on a PCI-e interface. To approximate a modern low-power server, we used a prototype Intel Pineview Atom-based system with two $1.8 \mathrm{GHz}$ cores, $2 \mathrm{~GB}$ of DRAM and an Intel X25-E SATA-based SSD. Unfortunately, production versions of this system were not available at the time we conducted this research: The prototype had only a $100 \mathrm{Mbps}$ Ethernet, which limited its performance, and the motherboard used low-efficiency voltage converters, which increased its power consumption. Between these extremes, we configured a "desktop" Core i7-based system with a single quad-core Core i7 860, 2 GB of DRAM, and 6 X25-E SATA drives. We attempted to balance this system by adding two SATA PCI-e cards because the motherboard supported only 4 SATA ports. We also reduced the power of this system by replacing the $40 \mathrm{~W}$ graphics card with a PCI card, and removed several extra DRAM chips for this particular experiment; through these efforts we reduced the desktop idle power to $45 \mathrm{~W}$. 
Table 1 shows that both the high-end server and desktop system could serve about $60,0001 \mathrm{~KB}$ queries per second from flash (queries and responses are over the network); the server's power draw was $194 \mathrm{~W}$ averaged over the length of the experiment, whereas the desktop's was far less at $98 \mathrm{~W}$. Thus, the desktop system was twice as energy-efficient as the server machine. In contrast, the Atom system could only provide 10,760 queries per second because it was limited by the $100 \mathrm{Mbps}$ Ethernet. Despite drawing only $22.3 \mathrm{~W}$, its limited performance placed its energy efficiency in between the other two systems.

There are two interesting observations to be made about these results. First, we note that the 60,000 queries/sec that both the server and the desktop provided is below saturation of the storage devices: The Fusion-io can provide 100,000 4 KB random reads per second and each X25-E can theoretically provide 35,000 $4 \mathrm{~KB}$ random reads based on filesystem benchmarking tools such as iozone [16] and fio [1]. Understanding this disparity is a topic of ongoing work. However, we note that when all values are retrieved from the filesystem buffer cache and avoid going to the device driver, the i7 systems can saturate a 1 Gbps network with requests, suggesting that the problem is specific to the I/O interface between our software and the flash devices.

Some of the performance bottlenecks may be fixed through software optimization while others may be more fundamentally related to the required processing or hardware architecture of the individual systems. None of the modern systems above are perfectly balanced in their use of CPU, memory and I/O, so we cannot make a strong conclusion about which platform will eventually be the most energy-efficient once any software bottlenecks are removed. But the main takeaway is that the lower-power systems (Atom and Desktop i7) are currently significantly more energy-efficient than traditional server architectures, and understanding the bottlenecks of each system should inform the design of future energy-efficient and balanced platforms for persistent key-value storage.

\subsection{Memory-bound workloads}

In the previous section, we discussed workloads whose working sets were large enough to require access to disks or flash, and that the computations on that data are simple enough to make the workload I/O-bound. In the next few sections, we explore some worstcase workloads designed to be more energy-efficient on traditional, high-power, high-speed systems than low-power, low-speed systems.

\subsubsection{Cache-bound Microbenchmark}

Workload description: We created a synthetic memory-bound benchmark that takes advantage of out-of-order execution and large caches. This benchmark repeatedly performs a matrix transpose multiplication, reading the matrix and vector data from memory and writing the result to memory. We chose matrix transpose specifically to have poor locality. The matrix data is in row-major format, which means that the transpose operation cannot sequentially stream data from memory. Each column of the matrix is physically separated in memory, requiring strided access and incurring more frequent cache evictions when the matrix does not fit entirely in cache.

The vector multiplications are data-independent to benefit from instruction reordering and pipelining, further biasing the workload in favor of modern high-speed, complex processors. We ran the

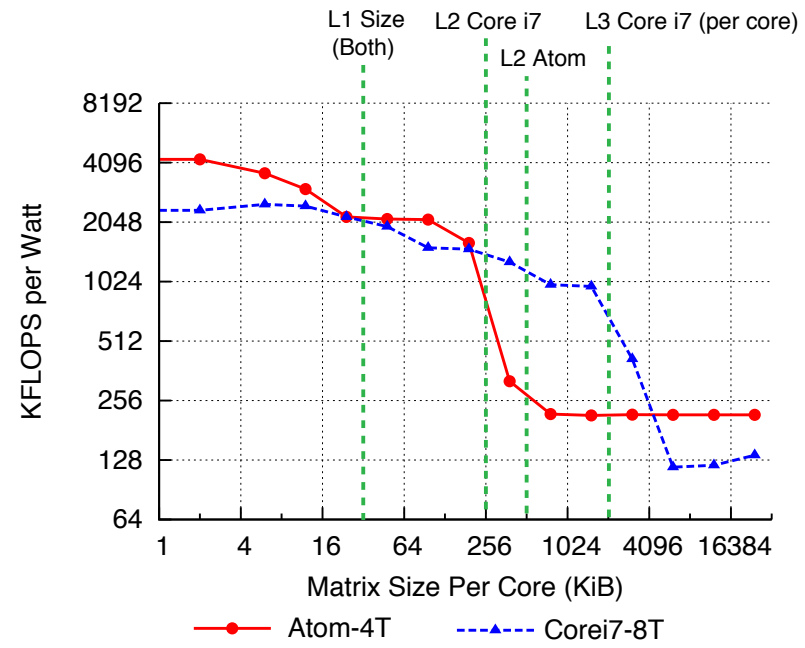

Figure 4: Efficiency vs. Matrix Size. Green vertical lines show cache sizes of each processor.

benchmark with various input matrix sizes. We estimate the metric of performance, FLOPS (floating point operations per second) as the number of multiply operations performed, though we note that this workload is more memory-intensive than CPU-intensive. ${ }^{2}$

Evaluation hardware: In this experiment, we compared only the i7-Desktop to our Atom chipset; the i7-Server's large fixed costs make it less efficient than the i7-Desktop in all cases. The i7Desktop operates 4 cores at a max of $2.8 \mathrm{GHz}$, though we used the Linux CPU ondemand scheduler to choose the appropriate speed for each workload. The i 7860 has a $32 \mathrm{~KB} \mathrm{L1}$ cache and a $256 \mathrm{~KB}$ L2 cache per core, and also has an $8 \mathrm{MB} \mathrm{L} 3$ cache shared across all 4 cores. We enabled two-way Hyper-threading (Simultaneous Multi-Threading) so that the system exposed 8 "processors" to the operating system. Finally, we removed all but one X25-E and one 2 GB DRAM DIMM to further reduce power. At idle, the power consumed by the machine was $40 \mathrm{~W}$ and at full load reached $130 \mathrm{~W}$.

The Atom's processor cores each have a $24 \mathrm{~KB} \mathrm{L1}$ data cache and a 512 KB L2 cache. Two-way hyper-threading was enabled, exposing 4 "processors" to the OS. At idle, the Atom system consumed $18 \mathrm{~W}$ and at full load would reach $29 \mathrm{~W}$.

Results: Figure 4 shows the energy efficiency (in KFLOPS/W) of our matrix multiply benchmark as a function of the size of the matrix being multiplied. When the matrix fits in the L1 data cache of both the i7-Desktop and the Atom, the Atom is roughly twice as efficient as the i7-Desktop. As the matrix size exceeds the L1 data cache, most memory accesses hit in L2 cache, and the efficiency drops by nearly a factor of two for both systems, with the Atom retaining higher energy efficiency.

The i7-Desktop's efficiency drops even further as the matrix size exhausts the $256 \mathrm{~KB}$ of L2 cache per core and accesses hit in L3. As the matrix size overflows the L2 cache on the Atom, most accesses then fall back to DRAM and efficiency remains flat thereafter. Meanwhile, the matrix size fits within the $8 \mathrm{MB}$ L3 cache of the i7. Once the matrix grows large enough, most of its accesses

\footnotetext{
${ }^{2}$ Comparing the FLOPS numbers here to those found in other CPU-intensive benchmarks such as in the Green500 competition will underestimate the actual computational capabilities of the platforms we measured, because this benchmark primarily measures memory $\mathrm{I} / \mathrm{O}$, not floating point operations.
} 


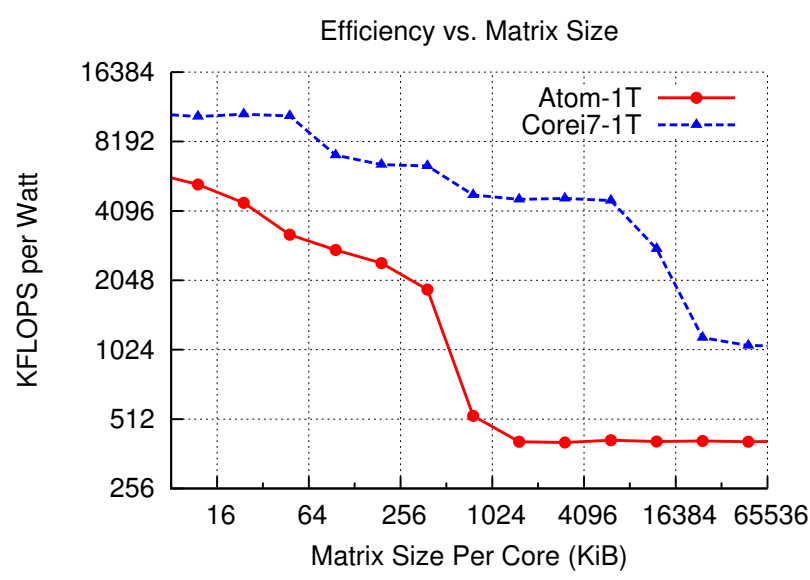

Figure 5: Efficiency vs. Matrix Size, Single Thread

then fall back to DRAM, and its energy efficiency drops below that of the Atom.

The main takeaway of this experiment is that when the working set fits in the same caches of each architecture, the Atom is up to twice as energy-efficient as the i7-Desktop. However, when the workload fits in the L2/L3 cache of the i7-Desktop but exhausts the Atom's on-die cache, the i7-Desktop is considerably more efficient, sometimes by a factor of four.

In other words, workloads that are cache-resident on a traditional system but not on a FAWN can be more efficient on the traditional system simply because of the amount of cache available on traditional systems.

The above experiment used OpenMP to run multiple threads simultaneously, eight threads on the i7-Desktop and four threads on the Atom. Running multiple threads is required to fully tax the CPU and memory systems of each node. We also ran the same experiment with one thread, to see how efficiency scales with load. Figure 5 shows that with one thread, the i7-Desktop is more efficient regardless of the size of the matrix.

This can be explained by fixed power costs. The i7-Desktop running one thread consumed $70 \mathrm{~W}$ (versus $40 \mathrm{~W}$ at idle), and the Atom running one thread consumed $20 \mathrm{~W}$ (versus $18 \mathrm{~W}$ at idle). The Atom platform we evaluated therefore has a large cost of not operating at full capacity. Its energy-proportionality is much worse than that of the i7-Desktop. Because the Atom was, at best, only twice as energy efficient as the i7-Desktop for this worst-case workload at $100 \%$ load, the inefficient chipset's power overhead dominates the CPU power and reduces the energy efficiency at low-load significantly. ${ }^{3}$

\subsubsection{JouleSort}

The above microbenchmark described a tightly controlled cache size-bound experiment showing that differences in cache sizes can significantly impact energy efficiency comparisons. But these discontinuities appear in more real world macrobenchmarks as well. More specifically, in this section we look at sorting many small

\footnotetext{
${ }^{3}$ In the case of our particular system, many of the fixed energy costs are due to non-"server" components: the GPU and video display circuitry, extra USB ports, and so on. Some components, however, such as the Ethernet port, cannot be eliminated. These same factors preclude the use of extremely low-power CPUs, as discussed in Section 2
}

records and describe our experiences competing for the 2010 10GB JouleSort competition. Our best system consists of a machine with a low-power server processor and five flash drives, sorting the 10GB dataset in 21.2 seconds $( \pm 0.227 \mathrm{~s})$ seconds with an average power of $104.9 \mathrm{~W}( \pm 0.8 \mathrm{~W})$. This system sorts the $10 \mathrm{~GB}$ dataset using only 2228 Joules $( \pm 12 \mathrm{~J})$, providing $44884( \pm 248)$ sorted records per Joule.

Our entry for the 10GB competition tried to use the most energyefficient platform we could find that could hold the dataset in memory to enable a one-pass sort. We decided to use a one-pass sort on this hardware over a two-pass sort on more energy efficient hardware (such as Intel Atom-based boards) after experimenting with several energy efficient hardware platforms that were unable to address enough memory to hold the 10GB dataset in memory. The low-power platforms we tested suffered from either a lack of $\mathrm{I} / \mathrm{O}$ capability or high, relative fixed power costs, both stemming from design decisions made by hardware vendors rather than being informed by fundamental properties of energy and computing.

Hardware: Our system uses an Intel Xeon L3426 $1.86 \mathrm{GHz}$ quad-core processor (with two hyperthreads per core, TurboBoostenabled) paired with 12GB of DDR3-1066 DRAM (2 DIMMS were 4GB modules and the other 2 DIMMS were $2 \mathrm{~GB}$ modules). The mainboard is a development board from 2009 based on an Intel 3420 chipset (to the best of our knowledge, this confers no specific power advantage compared to off-the-shelf versions of the board such as the Supermicro X8SIL-F or Intel S3420GPV Server Board), and we used a Prolimatech "Megahalems" fanless heatsink for the processor.

For storage, we use four SATA-based Intel X25-E flash drives (three had a $32 \mathrm{~GB}$ capacity and one had 64GB), and one PCIebased Fusion-io ioDrive (80GB). We use a 300W standard ATX power supply (FSP300) with a built-in and enabled cooling fan.

The storage devices were configured as follows: one small partition of a 32GB X25-E contained the OS. The other three X25-Es, the leftover portions of the OS disk, and the Fusion-IO (partitioned into three $10 \mathrm{~GB}$ partitions) were arranged in a single partition software RAID-0 configuration. Both the input and output file were located in a single directory within this partition. We used a Fusion-io in addition to 4 X25-Es because the SATA bus exists on the DMI bus with a bandwidth limitation of $10 \mathrm{Gbps}$ in theory and slightly less in practice. The Fusion-io was in a PCIe slot that is independent of the DMI bus and had a much higher bandwidth to the processor and memory system. Using both types of devices together therefore allowed us to more easily saturate the I/O and CPU capabilities of our system.

System power and software: The total power consumption of the system peaks at about $116 \mathrm{~W}$ during the experiment, but as mentioned below, averages about $105 \mathrm{~W}$ over the duration of the sort runs. While we do not have individual power numbers for each component during the experiment, the \{processor, DRAM, motherboard, power supply $\}$ combination consumes about $31 \mathrm{~W}$ at idle, the Fusion-io adds $6 \mathrm{~W}$ at idle, and each X25-E adds about $1 \mathrm{~W}$ to the idle power consumption for a total of $43 \mathrm{~W}$ at idle with all components attached.

All of our results are using Ubuntu Linux version 9.04 with kernel version 2.6.28 for driver compatibility with the Fusion-io device. We used ext4 with journaling disabled on the RAID-0 device. We use the gensort utility provided by the competition organizers (http://sortbenchmark.org) to create the $10^{8} 100$-byte records and use valsort to validate our final out- 


\begin{tabular}{lrrrr} 
& Time (s) & Power (W) & Energy (J) & SRecs/J \\
\hline Run 1 & 21.278 & 105.4 & 2242.5 & 44593 \\
Run 2 & 21.333 & 104.1 & 2219.8 & 45049 \\
Run 3 & 21.286 & 104.9 & 2232.6 & 44791 \\
Run 4 & 21.454 & 104.1 & 2233.7 & 44769 \\
Run 5 & 20.854 & 106.0 & 2211.5 & 45218 \\
\hline Avg & 21.241 & 104.9 & $\mathbf{2 2 2 8 . 0}$ & $\mathbf{4 4 8 8 4}$ \\
Error & 0.227 & 0.849 & 12.273 & 247.564
\end{tabular}

Table 2: Summary of JouleSort Experiment Results.

\begin{tabular}{lrrrr} 
& $\begin{array}{r}\text { In CPU } \\
\text { Util }\end{array}$ & $\begin{array}{r}\text { Out CPU } \\
\text { Util }\end{array}$ & $\begin{array}{r}\text { Input BW } \\
(\mathrm{MB} / \mathrm{s})\end{array}$ & $\begin{array}{r}\text { Output BW } \\
(\mathrm{MB} / \mathrm{s})\end{array}$ \\
\hline Run 1 & 343 & 628 & 973.71 & 1062 \\
Run 2 & 339 & 651 & 953.29 & 1074 \\
Run 3 & 339 & 613 & 971.82 & 1056 \\
Run 4 & 336 & 622 & 975.61 & 1050 \\
Run 5 & 343 & 646 & 976.56 & 1081 \\
\hline Avg & 340 & 632 & 970.198 & 1065 \\
Error & 3 & 16.078 & 9.626 & 12.759
\end{tabular}

Table 3: JouleSort CPU and bandwidth statistics.

put file. For sorting, we used a trial version of NSort software (http://www.ordinal.com).

Results: Our results are summarized in the Table 2. Our system improves upon the January 2010 Daytona winner by nearly a factor of two, and also improves upon the January 2010 Indy winner by $26 \%$ [5]. The January 2010 Indy winner group's more recent entry closes this gap to 5\% for the Indy designation and $12 \%$ for the Daytona designation.

We log the statistics provided by NSort for comparison with [9]. Table 3 summarizes the information (Utilization measured out of a total of $800 \%$ and bandwidth measured in terms of $\mathrm{MB} / \mathrm{s}$ for reading and writing the data).

Experiences: Our submission used a server-class system as opposed to a low-power component system like the Intel Atom. The dominating factor in this choice was the ability of our server system to hold the entire 10GB dataset in DRAM to enable a one-pass sort-in this case, the energy efficiency benefits of performing a one-pass sort outweighed the hardware-based energy efficiency of low-power platforms that must perform a two-pass sort. Our submission tried to use the most energy-efficient platform we could find that allowed for a one-pass sort, and this turned out to use the low-frequency Xeon platform described above. Below, we describe some details about what other systems we tried before settling on the entry system described above.

Alternative Platforms: We tried several alternative low-power configurations based on the Intel Atom as part of our research into the FAWN approach [2]. In particular, we began with the Zotac Ion board based on an Intel Dual-core Atom 330 (also used by Beckmann et. al) paired with 4 Intel X25-E drives. Without any special software tweaking, we were able to get approximately 35000 SRecs/J at an average power of about $33 \mathrm{~W}$. We also tried to use the NVidia GPU available on the Ion to do a portion of the sorting, but found that the I/O was the major bottleneck regardless.

We also experimented with a single core Atom board by Advantech paired with 1 X25-E, and a dual-core Atom Pineview develop-

\begin{tabular}{lrr} 
Workload & i7-Desktop & Atom \\
\hline SHA-1 & & \\
MB/s & 360 & 107 \\
Watts & 75 & 19.1 \\
MB/J & 4.8 & 5.6 \\
\hline SHA-1 multi-process & & \\
MB/s & 1187 & 259 \\
Watts & 117 & 20.7 \\
MB/J & 10.1 & 12.51 \\
\hline$R S A$ & & \\
Sign/s & 8748 & 1173.5 \\
Verify/s & 170248 & 21279.0 \\
Watts & 124 & 21.0 \\
Sign/J & 70.6 & 55.9 \\
Verify/J & 1373 & 1013
\end{tabular}

Table 4: Encryption Speed and Efficiency

ment board with two X25-Es. These boards were both lower power than the Zotac Ion-the Pineview board moved from a three-chip to a two-chip solution, placing the graphics and memory controllers on-die, thus reducing chipset power slightly. We also tried attaching a Fusion-io board to a dual-core Atom system, but because the Fusion-io currently requires significant host processing and memory, the Atom could not saturate the capabilities of the drive and so was not currently a good fit.

\subsection{CPU-bound workloads}

The memory-bound workloads in the previous section required frequent memory accesses per computation across a large dataset Next, we look at a CPU-intensive task: cryptography. Table 4 shows several assembly-optimized OpenSSL speed benchmarks on the i7-Desktop and Atom systems described above. On SHA-1 workloads, we find that the Atom-based platform is slightly more efficient in terms of work done per Joule than the i7-Desktop architecture, and for RSA sign/verify, the reverse is true.

This flip in efficiency appears to be due to the optimization choices made in the assembly code versions of the algorithms. The OpenSSL "C" implementations of both SHA-1 and RSA are both more efficient on the Atom; we hypothesize that the asm version is tuned for high-performance CPUs. The SHA-1 assembly implementation, in contrast, was recently changed to use instructions that also work well on the Atom, and so its efficiency again exceeds that of the i7-Desktop. These results suggest that, first, CPU-bound operations can be as or more efficient on low-power processors, and second, they underscore that nothing comes for free: code must sometimes be tweaked, or even rewritten, to run well on these different architectures.

\subsection{Limitations}

FAWN and other low-power many-core cluster architectures may be unsuited for some datacenter workloads. These workloads can be broadly classified into two categories: latency-sensitive, nonparallelizable workloads and memory-hungry workloads. 


\subsubsection{Latency-sensitive, non-parallelizable}

As mentioned previously, the FAWN approach of reducing speed for increased energy efficiency relies on the ability to parallelize workloads into smaller discrete chunks, using more nodes in parallel to meet performance goals; this is also known as the scale-out approach. Unfortunately, not all workloads in data-intensive computing are currently amenable to this type of parallelism.

Consider a workload that requires encrypting a $64 \mathrm{MB}$ chunk of data within 1 second, and assume that a traditional node can optimally encrypt at $100 \mathrm{MB} / \mathrm{sec}$ and a FAWN node at $20 \mathrm{MB} / \mathrm{sec}$. If the encryption cannot be parallelized, the FAWN node will not encrypt data fast enough to meet the strict deadline of 1 second, whereas the traditional node would succeed. Note that if the fastest system available was insufficient to meet a particular latency deadline, parallelizing the workload here would no longer be optional for either architecture. Thus, the move to many-core architectures (with individual core speed reaching a plateau) poses a similar challenge of requiring application parallelism. ${ }^{4}$

\subsubsection{Memory-hungry workloads}

Workloads that demand large amounts of memory per process are another difficult target for FAWN architectures. We examined a workload derived from a machine learning application that takes a massive-data approach to semi-supervised, automated learning of word classification. The problem reduces to counting the number of times each phrase, from a set of thousands to millions of phrases, occurs in a massive corpus of sentences extracted from the Web. Our results are promising but challenging. FAWN converts a formerly I/O-bound problem into a memory size-bound problem, which requires algorithmic and implementation attention to work well. The Alix $3 \mathrm{c} 2$ nodes can grep for a single pattern at $25 \mathrm{MB} / \mathrm{sec}$, close to the maximum rate the $\mathrm{CF}$ can provide. However, searching for thousands or millions of phrases with the naive Aho-Corasick algorithm in grep requires building a DFA data structure that requires several gigabytes of memory. Although this structure fit in the memory of conventional architectures equipped with 8-16 GB of DRAM, it quickly exhausted the $256 \mathrm{MB}$ of DRAM on each individual FAWN node.

To enable this search to function on a node with tight memory constraints, we optimized the search using a rolling hash function and large bloom filter to provide a one-sided error grep (false positive but no false negatives) that achieves roughly twice the energy efficiency (bytes per second per Watt) as a conventional node [20].

However, this improved efficiency came at the cost of considerable implementation effort. Our experience suggests that efficiently using FAWN nodes for some scan-based workloads will require the development of easy-to-use frameworks that provide common, heavily-optimized data reduction operations (e.g., grep, multi-word grep, etc.) as primitives. This represents an exciting avenue of future work: while speeding up hardware is difficult, programmers have long excelled at finding ways to optimize CPU-bound problems.

An interesting consequence of this optimization was that the same techniques to allow the problem to fit in DRAM on a FAWN

\footnotetext{
${ }^{4}$ Indeed, this challenge is apparent to the designers of next-generation cryptographic algorithms: Several of the entrants to the NIST SHA-3 secure hash competition include a hash-tree mode for fast, parallel cryptographic hashing. The need for parallel core algorithms continues to grow as multi- and many-core approaches find increased success. We believe this general need for parallel algorithms will help make the FAWN many-core approach even more feasible.
}

node drastically improved cache performance on more conventional architectures: We were able to apply the techniques we developed to double the speed of virus scanning on desktop machines [8].

\subsection{Lessons Learned}

In this section, we summarize some of the lessons we have learned about applying FAWN to a broader set of workloads. We break down these lessons into two different categories: software challenges and hardware challenges.

\subsubsection{Software Challenges}

A recurring theme that arises in working with FAWN systems is that existing software often does not run as well on FAWN node platforms. When deploying out-of-the-box software on FAWN and finding poor efficiency results, it is critically important to identify precisely the characteristics of the workload or the software that reduce efficiency. For example, many applications are becoming increasingly memory hungry as server-class hardware makes more memory per node available. As we have shown, the working set size of a cache- or memory-bound application can be an important factor in the FAWN vs. traditional comparison. If these applications cannot reduce their working set size, this is a fundamental limitation that FAWN systems may not overcome. Fortunately, many algorithmic changes to software can improve memory efficiency to the point where the application's performance on a FAWN platform significantly increases. This emphasizes that writing efficient software on top of efficient hardware has a large role in improving energy efficiency.

Memory efficiency is not the only software challenge to overcome when considering FAWN systems. By shrinking the CPUI/O gap, more balanced systems may become CPU-bound when processing I/O by exposing previously unimportant design and implementation inefficiencies. In our work, for example, we have observed that the Linux block layer-designed and optimized for rotating media-imposes high per-request overhead that makes it difficult to saturate a modern flash drive using a single or dual-core Atom processor. We have made several kernel changes to the block layer, such as improving hardware interrupt handling and eliminating the entropy pool calculation on each block request, in an effort to eliminate CPU bottlenecks. While we have been moderately successful (we have improved I/O throughput by over $60 \%$ on an Atom platform), we continue to explore more software optimizations to see if we can make better use of the I/O capability available on newer Atom boards with more SATA ports. Additionally, Linux versions after 2.6.28 include several small modifications to the block layer that better support flash SSDs, which should improve performance for both low-power and traditional systems.

\subsubsection{Hardware Challenges}

Many of today's hardware platforms appear capable of further improvements to energy efficiency, but are currently limited in practice due to several factors, many of which are simply due to choices made by hardware vendors of low-power platforms:

High idle/fixed cost power: The boards we have used all idled at $15-20 \mathrm{~W}$ even though their peak is only about $10-15 \mathrm{~W}$ higher. Fixed costs affect both traditional processors and low-power CPUs alike, but the proportionally higher fixed-cost to peak-power ratio 
on available Atom platforms diminishes some of the benefits of the low-power processor.

IO and bus limitations: When exploring the sort benchmark, we found it difficult to find systems that provided sufficient I/O to saturate the processor. Most Atom boards provided only two SATA drive connectors. While Supermicro recently released one with six ports, they were connected to the CPU over a bandwidth-limited DMI bus; this bus provides 10Gbps in each direction, which can support only four X25-E SSDs reading at $250 \mathrm{MB} /$ second. These limitations may reflect the fact that these processors are not aimed at the server market in which I/O typically receives more emphasis.

The market for ultra low power server systems has greatly expanded over the last several years, with companies such as SeaMicro, Marvell, Calxeda and ZT Systems all producing lowpower datacenter computing platforms. We expect many of the non-fundamental hardware challenges to disappear as competition drives further innovation, but many of the fundamental challenges (e.g., the unavoidable fixed power costs posed by having an onboard Ethernet chip or I/O controllers) will always play a large role in determining the most efficient balance and absolute speed of CPU and I/O on an energy-efficient platform.

\section{IMPLICATIONS AND OUTLOOK}

In Section 2, we outlined several power scaling trends for modern computer systems. Our workload evaluation in the previous section suggested that these trends hold for CPU in real systems-and that, as a result, using slower, simpler processors represents an opportunity to reduce the total power needed to solve a problem if that problem can be solved at a higher degree of parallelism.

In this section, we draw upon the memory scaling trends we discussed to present a vision for a future FAWN system: Individual "nodes" consisting of a single CPU chip with a modest number of relatively low-frequency cores, with a small amount of DRAM stacked on top of it, connected to a shared interconnect. This architecture is depicted in Figure 6. The reasons for such a choice are several:

Many, many cores: The first consequence of the scaling trends is clear: A future energy-efficient system for data-intensive workloads will have many, many cores, operating at quite modest frequencies. The limits of this architecture will be the degree to which algorithms can be parallelized (and/or load-balanced), and the static power draw imposed by CPU leakage currents and any hardware whose power draw does not decrease as the size and frequency of the cores decrease.

However, the move to many-core does not imply that individual chips must have modest capability. Indeed, both Intel and startups such as Tilera have demonstrated prototypes with $48-100$ cores on a single chip. Such a design has the advantage of being able to cheaply interconnect cores on the same chip, but suffers from limited off-chip IO and memory bandwidth compared to the amount of CPU on chip.

Less memory, stacked: We chose a stacked DRAM approach because it provides three key advantages: Higher DRAM bandwidth, lower DRAM latency (perhaps half the latency of a traditional DIMM bus architecture) and lower DRAM power draw. The disadvantage is the limited amount of memory available per chip. Using the leading edge of today's DRAM technologies, an 8Gbit DRAM chip could be stacked on top of a small processor; 1GB of DRAM for a single or dual-core Atom is at the low end of an acceptable

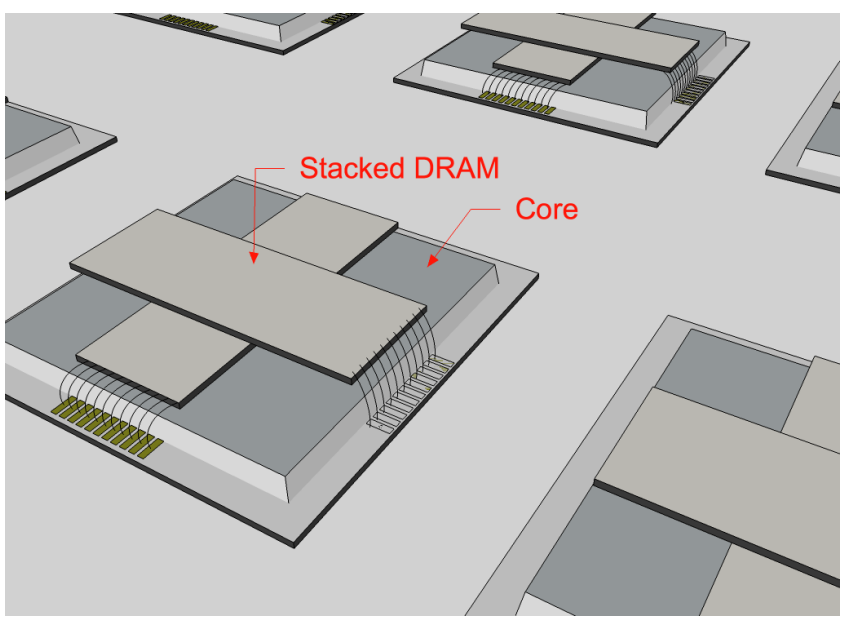

Figure 6: Future FAWN vision: Many-core, low-frequency chip with stacked DRAM per core.

amount of memory for many workloads. From the matrix multiplication workload in the previous section, we expect that this decision will result in a similar efficiency "flip-flop": Workloads that fit in memory on a single FAWN node with 1 GB of DRAM would run much more efficiently than they would on a comparable large node, but the FAWN node would be less efficient for the range of problems that exceed 1GB but are small enough to fit in DRAM on a more conventional server.

However, the challenges posed by this architecture raise several issues:

Optimization back in vogue: Software efficiency was once a community focus: ekeing every last drop of performance or resource from a system was a laudable goal. With the rapid growth of data-intensive computing and a reliance on Moore's law, today's developers are less likely to optimize resource utilization, instead focusing on scalability at the detriment of node efficiency [3]. Instead, the focus has been on scalability, reliability, manageability, and programmability of clusters. With a FAWN-like architecture, each node has fewer resources, making the job of the programmers harder. Our prior work has shown that the limited amount of memory per node has required the design of new algorithms [20] and careful balance of performance and memory footprint for inmemory hashtables [2]. These difficulties are compounded by the higher expected node count in FAWN architectures-not only does resource utilization become more important, these architectures will further stress scalability, reliability, and manageability.

Heterogeneity: The existence of problems for which conventional server architectures still reign suggests that clusters must embrace heterogeneity in computing resources. Today's large-scale systems already must deal with heterogeneity because of arbitrary node failures and cluster purchasing schedules, but the existence of more energy-efficient, slower nodes will require that application and infrastructure software treat them as first-class resources with energy metrics playing a larger role in resource allocation decisions.

Metrics: We have so far evaluated energy efficiency in work done per Joule, which combines performance and power together as the only metrics. However, energy's impact on data-intensive computing is more broad-recent work has shown that platforms such as the Atom have other externalities, such as increased variability 
and latency, which affects service level agreements and other such quality of service metrics [21]. Indeed, we believe a better, general metric to focus on improving is "Quality of Service per Joule" $(\mathrm{QoS} / \mathrm{J})$, which captures average, worst-case and baseline performance requirements in one metric. A focus of our ongoing work is to improve Quality of Service per Joule without microarchitectural redesigns (when possible), and also to carefully devise metrics to properly capture and quantify these more difficult externalities.

\section{RELATED WORK}

FAWN follows in a long tradition of ensuring that systems are balanced in the presence of scaling challenges and of designing systems to cope with the performance challenges imposed by hardware architectures.

System Architectures: JouleSort [23] is a recent energy efficiency benchmark; its authors developed a SATA disk-based "balanced" system coupled with a low-power (34 W) CPU that significantly out-performed prior systems in terms of records sorted per joule. The results from this earlier work match our own in finding that a low-power CPU is easier to balance against I/O to achieve efficient sorting performance.

More recently, several projects have begun using low-power processors for datacenter workloads to reduce energy consumption [7, 19, 11, 27, 14, 18]. The Gordon [7] hardware architecture argues for pairing an array of flash chips and DRAM with lowpower CPUs for low-power data intensive computing. A primary focus of their work is on developing a Flash Translation Layer suitable for pairing a single CPU with several raw flash chips. Simulations on general system traces indicate that this pairing can provide improved energy efficiency. CEMS [14], AmdahlBlades [27], and Microblades [18] also leverage low-cost, low-power commodity components as a building block for datacenter systems, similarly arguing that this architecture can provide the highest work done per dollar and work done per joule. Microsoft has recently begun exploring the use of a large cluster of low-power systems called Marlowe [19]. This work focuses on taking advantage of the very low-power sleep states provided by this chipset (between 2-4 W) to turn off machines and migrate workloads during idle periods and low utilization, initially targeting the Hotmail service. We believe these advantages would also translate well to FAWN, where a lull in the use of a FAWN cluster would provide the opportunity to significantly reduce average energy consumption in addition to the already-reduced peak energy consumption that FAWN provides. Dell recently begun shipping VIA Nano-based servers consuming 20-30 W each for large webhosting services [11].

Considerable prior work has examined ways to tackle the "memory wall." The Intelligent RAM (IRAM) project combined CPUs and memory into a single unit, with a particular focus on energy efficiency [6]. An IRAM-based CPU could use a quarter of the power of a conventional system to serve the same workload, reducing total system energy consumption to $40 \%$. FAWN takes a thematically similar view - placing smaller processors very near flash-but with a significantly different realization. Notably, our vision for a future FAWN with stacked DRAM grows closer to the IRAM vision, though avoiding the embedded DRAM that plagued the IRAM implementation. Similar efforts, such as the Active Disk project [22], focused on harnessing computation close to disks. Schlosser et al. proposed obtaining similar benefits from coupling MEMS with CPUs [24].
Sleeping: A final set of research examines how and when to put machines to sleep. Broadly speaking, these approaches examine the CPU, the disk, and the entire machine. We believe that the FAWN approach compliments them well. Because of the data-intensive focus of FAWN, we focus on several schemes for sleeping disks: Hibernator [31], for instance, focuses on large but low-rate OLTP database workloads (a few hundred queries/sec). Ganesh et al. proposed using a log-structured filesystem so that a striping system could perfectly predict which disks must be awake for writing [12]. Finally, Pergamum [26] used nodes much like our FAWN nodes to attach to spun-down disks for archival storage purposes, noting that the nodes consume much less power when asleep. The system achieved low power, though its throughput was limited by the nodes' Ethernet.

\section{CONCLUSION}

This paper presented the computing trends that motivate our Fast Array of Wimpy Nodes (FAWN) architecture, focusing on the continually increasing CPU-Memory and CPU-I/O gap and the superlinear increase in power vs. single-component speed. Our evaluation of a variety of workloads, from worst-case seek-bound I/O workloads to pure CPU or memory benchmarks, suggests that overall, lower frequency nodes can be substantially more energy efficient than more conventional high-performance CPUs. The exceptions lie in problems that cannot be parallelized or whose working set size cannot be split to fit in the cache or memory available to the smaller nodes. These trends point to a realistic, but difficult, path for energy efficient computing: Accepting tight constraints on per-node performance, cache, and memory capacity, together with using algorithms that scale to an order of magnitude more processing elements. While many data-intensive workloads may fit this model nearly out-of-the-box, others may require substantial algorithmic and implementation changes.

\section{Acknowledgments}

We thank Jason Campbell for feedback that improved the clarity of this paper. We would also like to thank Ken Mai for his help with understanding memory trends. Amar Phanishayee contributed extensively to the design and implementation of the FAWN-KV key value system used for several of the experiments described in Section 3.3. We would also like to thank Kanat Tangwongsan for his aid in developing the matrix multiply benchmark used in this work. This work was supported in part by gifts from Network Appliance, Google, and Intel Corporation, and by grants CNS-0619525 and CCF-0964474 from the National Science Foundation.

\section{References}

[1] Flexible I/O Tester. http://freshmeat.net/projects/ fio/.

[2] David G. Andersen, Jason Franklin, Michael Kaminsky, Amar Phanishayee, Lawrence Tan, and Vijay Vasudevan. FAWN: A fast array of wimpy nodes. In Proc. 22nd ACM Symposium on Operating Systems Principles (SOSP), Big Sky, MT, October 2009.

[3] Eric Anderson and Joseph Tucek. Efficiency matters! In Proc. HotStorage, Big Sky, MT, October 2009.

[4] Luiz André Barroso and Urs Hölzle. The case for energy-proportional computing. Computer, 40(12):33-37, 2007. 
[5] Andreas Beckmann, Ulrich Meyer, Peter Sanders, and Johannes Singler. Energy-efficient sorting using solid state disks. http:// sortbenchmark.org/ecosort_2010_Jan_01.pdf, 2010.

[6] W. Bowman, N. Cardwell, C. Kozyrakis, C. Romer, and H. Wang. Evaluation of existing architectures in IRAM systems. In Workshop on Mixing Logic and DRAM, 24th International Symposium on Computer Architecture, June 1997.

[7] Adrian M. Caulfield, Laura M. Grupp, and Steven Swanson. Gordon: Using flash memory to build fast, power-efficient clusters for data-intensive applications. In 14th International Conference on Architectural Support for Programming Languages and Operating Systems (ASPLOS '09), March 2009.

[8] Sang Kil Cha, Iulian Moraru, Jiyong Jang, John Truelove, David Brumley, and David G. Andersen. SplitScreen: Enabling efficient, distributed malware detection. In Proc. 7th USENIX NSDI, San Jose, CA, April 2010.

[9] John D. Davis and Suzanne Rivoire. Building energy-efficient systems for sequential workloads. Technical Report MSR-TR-2010-30, Microsoft Research, March 2010.

[10] Jeffrey Dean and Sanjay Ghemawat. MapReduce: Simplified data processing on large clusters. In Proc. 6th USENIX OSDI, San Francisco, CA, December 2004.

[11] Dell fortuna. http://www1.euro.dell.com/content/ topics/topic.aspx/emea/corporate/pressoffice/ 2009/uk/en/2009_05_20_brk_000, 2009.

[12] Lakshmi Ganesh, Hakim Weatherspoon, Mahesh Balakrishnan, and Ken Birman. Optimizing power consumption in large scale storage systems. In Proc. HotOS XI, San Diego, CA, May 2007.

[13] Kathy Gray. Port deal with Google to create jobs. The Dalles Chronicle, http://www.gorgebusiness.com/2005/ google. htm, February 2005.

[14] James Hamilton. Cooperative expendable micro-slice servers (CEMS): Low cost, low power servers for Internet scale services. http://mvdirona.com/jrh/TalksAndPapers/ JamesHamilton_CEMS.pdf, 2009.

[15] Penryn Press Release. http: //www. intel.com/pressroom/ archive/releases/20070328fact.htm.

[16] Filesystem Benchmark. http://www. iozone.org.

[17] Randy H. Katz. Tech titans building boom. IEEE Spectrum, February 2009.

[18] Kevin Lim, Parthasarathy Ranganathan, Jichuan Chang, Chandrakant Patel, Trevor Mudge, and Steven Reinhardt. Understanding and designing new server architectures for emerging warehouse-computing environments. In International Symposium on Computer Architecture (ISCA), Beijing, China, June 2008.

[19] Peering into future of cloud computing. http://research. microsoft.com/en-us/news/features/ccf-022409. aspx, 2009.

[20] Iulian Moraru and David G. Andersen. Exact pattern matching with feed-forward bloom filters. In Proceedings of the Workshop on Algorithm Engineering and Experiments (ALENEX11), ALENEX 2011. Society for Industrial and Applied Mathematics, 2011.

[21] Vijay Janapa Reddi, Benjamin Lee, Trishul Chilimbi, and Kushagra Vaid. Web search using small cores: Quantifying the price of efficiency. Technical Report MSR-TR-2009-105, Microsoft Research, August 2009.

[22] Erik Riedel, Christos Faloutsos, Garth A. Gibson, and David Nagle. Active disks for large-scale data processing. IEEE Computer, 34(6):68-74, June 2001.

[23] Suzanne Rivoire, Mehul A. Shah, Parthasarathy Ranganathan, and Christos Kozyrakis. JouleSort: A balanced energy-efficient benchmark. In Proc. ACM SIGMOD, Beijing, China, June 2007.

[24] Steven W. Schlosser, John Linwood Griffin, David F. Nagle, and Gregory R. Ganger. Filling the memory access gap: A case for on-chip magnetic storage. Technical Report CMU-CS-99-174, Carnegie Mellon University, November 1999.

[25] Seamicro. http://www. seamicro.com, 2010.

[26] Mark W. Storer, Kevin M. Greenan, Ethan L. Miller, and Kaladhar Voruganti. Pergamum: Replacing tape with energy efficient, reliable, disk-based archival storage. In Proc. USENIX Conference on File and Storage Technologies (FAST 2008), San Jose, CA, February 2008.

[27] Alex Szalay, Gordon Bell, Andreas Terzis, Alainna White, and Jan Vandenberg. Low power Amdahl blades for data intensive computing, 2009.

[28] Niraj Tolia, Zhikui Wang, Manish Marwah, Cullen Bash, Parthasarathy Ranganathan, and Xiaoyun Zhu. Delivering energy proportionality with non energy-proportional systems - optimizing the ensemble. In Proc. HotPower, San Diego, CA, December 2008.

[29] .NET Power Meter. http: / / watt supmeters . com.

[30] Mark Weiser, Brent Welch, Alan Demers, and Scott Shenker. Scheduling for reduced CPU energy. In Proc. 1st USENIX OSDI, pages 13-23, Monterey, CA, November 1994.

[31] Qingbo Zhu, Zhifeng Chen, Lin Tan, Yuanyuan Zhou, Kimberly Keeton, and Jon Wilkes. Hibernator: Helping disk arrays sleep through the winter. In Proc. 20th ACM Symposium on Operating Systems Principles (SOSP), Brighton, UK, October 2005. 